\title{
Sex-specific association between cardiac workload, arterial stiffness, peripheral vascular calcification and body composition: The Gambian bone and muscle ageing study
}

\author{
A. Zengin ${ }^{1}$, M. Ó Breasail ${ }^{2}$, C. M. Parsons ${ }^{3}$, L.M. Jarjou ${ }^{4}$, R.E. Janha ${ }^{4}$, A. Prentice ${ }^{2,4}$, \\ C Cooper ${ }^{3}$, Peter R Ebeling ${ }^{1}$ and K. A. Ward ${ }^{3,4}$ \\ ${ }^{1}$ Department of Medicine, School of Clinical Sciences at Monash Health, Monash University, Australia, \\ ${ }^{2}$ MRC Nutrition and Bone Health Research Group, Cambridge, UK, \\ ${ }^{3}$ MRC Lifecourse Epidemiology Unit, University of Southampton, Southampton, UK and \\ ${ }^{4}$ MRC Unit The Gambia, London School of Hygiene and Tropical Medicine, Banjul, The Gambia
}

Increased longevity, nutrition and economic transition and increased urbanisation are leading to increased burden of noncommunicable diseases (NCDs) of ageing in Africa. Characterisation of the association between hypertension and body composition and muscle function may help identify preventative strategies for cardiovascular disease and sarcopenia in Sub-Saharan African countries such as The Gambia ${ }^{(1,2)}$. Therefore, the aims of this study were: to investigate whether there are sex differences in the associations between markers of cardiovascular disease (CVD) and body composition and explore whether CVD risk factors are predictive of poor physical function.

The Gambian Bone and Muscle Ageing Study (GamBAS) is a longitudinal prospective observational study in 249 women and 239 men from rural Gambia aged 40-90 years ${ }^{(1)}$. Each participant had a whole body scan by dual energy x-ray absorptiometry (DXA), and body composition outcomes obtained were: total fat mass (FM, kg), total lean mass (kg), android fat mass (AFM, kg) and gynoid fat mass (GFM, kg). Supine blood pressure and heart rate were measured and used to calculate rate pressure product and pulse pressure, both were log transformed to normalise distributions and to allow expression of the results as percent difference per $10 \%$ change. Peripheral quantitative computed tomography (pQCT) was used to assess muscle cross-sectional area and peripheral vascular calcification (PVC) in the lower leg (66\% tibia). Handgrip strength $(\mathrm{kg})$ was measured using a hand dynamometer. Jumping mechanography measured leg muscle power $(\mathrm{kW})$ following a 2-legged jump and a chair rise test (CRT), 3 repeated chair stands. Relationships between rate pressure product and pulse pressure with body composition/physical function parameters were explored using linear regression with adjustment for age and height, and sex-interactions were tested. Results are presented as beta [95\% CI].

There were sex differences in the associations between rate pressure product and body fat compartments (total, android and gynoid). In men, every $10 \%$ increase in rate pressure product was positively associated with fat mass at the: total body ( 9.3 $\left.[6.3,12.4]^{\%}\right)$, android $(11.1[7.0,15.1] \%)$ and gynoid regions $(7.6[4.8,10.3] \%)$. Conversely, in women no significant associations were found. No consistent sex differences were observed between fat mass and pulse pressure or presence of PVC. For muscle function, there were negative associations between pulse pressure and handgrip strength $(-0.48,[-0.79,-0.17] \%)$, CRT power $(-0.16$ $[-0.26,-0.06] \%)$ and jump power $\left(-0.027[-0.58,0.004]^{\%}\right)$ in men. Men with PVC also had lower grip strength $\left(-2.27,[-4.15,-0.40]^{\%}\right)$ and jump power $(-0.17[-0.36,0.02] \%)$. In contrast, no sex differences in the relationship between cardiac markers and lean mass or muscle cross-sectional area were found.

Men had negative associations between CVD parameters, body fat and physical function, while women did not. In this population, targeting improvements in cardiovascular health, may have beneficial effects on physical function and adiposity in men.

\section{References}

1. Zengin A, Fulford AJ, Sawo Y, et al. (2017) Front Endocrinol 8:219.

2. Zengin A, Jarjou LM, Prentice A, et al. (2018) J Cachexia Sarcopenia Muscle 9(5):920-8. 